MODELING, IDENTIFICATION AND CONTROL, 1992, VOL. 13, NO. 1, 15-23

doi:10.4173/mic.1992.1.2

\title{
On the location of LQ-optimal closed-loop poles
}

\section{DAVID DI RUSCIO†}

Keywords: Linear optimal control, eigenvalues, inequalities, pole placements.

\begin{abstract}
Inequalities which bound the closed-loop eigenvalues in an LQ-optimal system are presented. It is shown that the eigenvalues are bounded by two half circles with radii $r_{1}$ and $r_{2}$ and centre at $-\alpha \leqslant 0$, where $\alpha=0$ is the imaginary axis, and that the imaginary parts of these eigenvalues are bounded from up and below by two lines parallel to the real axis.
\end{abstract}

\section{Introduction}

Application of the LQ approach to regulator design involves choosing the state and control input weighting matrices, $\mathbf{Q}$ and $\mathbf{P}$ that provide satisfactory closed-loop performance. The closed-loop performance is related to the locations of the closed-loop eigenvalues. Therefore, the relation between the quadratic weights and the poles of the closed-loop system is of interest. This problem has been solved for a second order system, (Di Ruscio and Balchen, 1990). In the general case, very little is known about these relations.

However, instead of having exact knowledge of their positions, it may be sufficient to know that the poles are located in a bounded region of the left half $s$-plane. This paper is concerned with the problem of determining the region where the closed-loop eigenvalues are located.

The paper will show that for a suitable choice of performance weighting matrices, the eigenvalues are bounded by two half circles with radii $r_{1}$ and $r_{2}$ and centre at

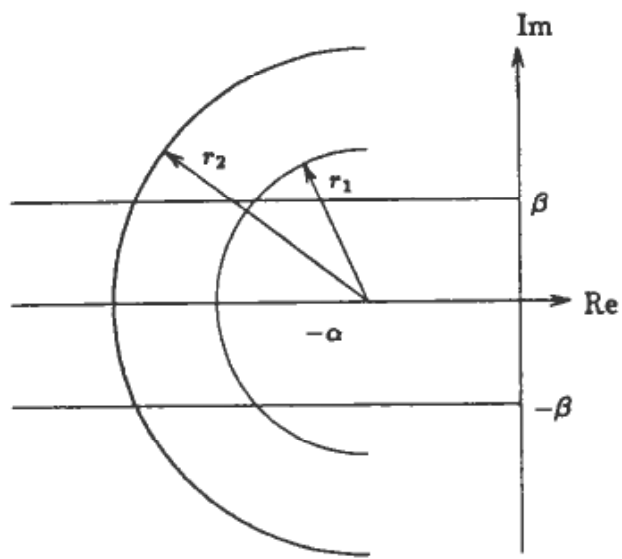

Figure 1. Region where the closed-loop poles are located.

Received 11 November 1991.

$\dagger$ Division of Engineering Cybernetics, Norwegian Institute of Technology, N-7034 Trondheim, Norway.

This paper was presented at the 30th IEEE Conference on Decision and Control, Brighton, England, 11-13 December 1991. Reprinted with permission from the IEEE. 
$-\alpha \leqslant 0$, where $\alpha=0$ is the imaginary axis, and that the imaginary parts of these eigenvalues are bounded from up and below by two lines parallel to the real axis. This region is illustrated in Figure (1).

The paper is organised as follows: Section 2 presents the problem definitions. The problem solution is stated in Section 3 and some concluding remarks follow in Section 4.

\section{Problem formulation}

Consider the linear, time-invariant, dynamic system

$$
\dot{\boldsymbol{x}}=A \boldsymbol{x}+B \boldsymbol{u}
$$

where $(A, B)$ is a stabilizable pair, and the exponentially weighted quadratic objective functional of long or infinite settling time

$$
J=\frac{1}{2} \int_{0}^{\infty} \exp (2 \alpha t)\left(x^{\mathrm{T}} Q x+u^{\mathrm{T}} P u\right) d t
$$

where $\alpha \geqslant 0$ is a real number, $\boldsymbol{x}$ is an $\boldsymbol{n}$-dimensional state vector, $\boldsymbol{u}$ is an $\boldsymbol{r}$-dimensional control input vector, $A$ and $B$ are constant matrices of appropriate dimensions and $Q$ and $\mathrm{P}$ are $n \times n$ symmetrical and $r \times \boldsymbol{r}$ positive definite matrices respectively. It is assumed that $\left(Q^{\frac{1}{2}}, A\right)$ is a detectable pair. The optimal control that minimizes criterion (2) and the corresponding closed-loop system is given by

$$
\begin{aligned}
& u=G x=-P^{-1} B^{\mathrm{T}} R x \\
& \dot{x}=(A+B G) x=(A-H R) x
\end{aligned}
$$

where $\boldsymbol{H}$ is given by (6) and $\boldsymbol{R}$ is a solution to the algebraic Riccati equation (ARE)

$$
\begin{aligned}
-\dot{R} & =A^{\mathrm{T}} R+R A-R H R+2 \alpha R+Q=0 \\
H & =B P^{-1} B^{\mathrm{T}}
\end{aligned}
$$

The closed-loop system (4) is $\alpha$ stable, i.e. all eigenvalues with real part less than $-\alpha$.

The problem investigated in this paper is to determine the region which the LQoptimal closed-loop poles are located.

\section{Main results}

The main results in this section are stated in Subsections 3.1, 3.2 and 3.3. Subsection 3.1 deals with the imaginary part, Subsection 3.2 considers the magnitude and Subsection 3.3 is concerned with the real part of the closed-loop eigenvalues.

\subsection{Imaginary parts of the closed loop eigenvalues}

The main result in this section is presented in the following theorem.

\section{Theorem 1}

The imaginary parts of the LQ-optimal closed-loop eigenvalues, $\left(s_{i}=x_{i}+j y_{i}\right.$, $i=1, \ldots, n)$, are bounded, from up and below, by

where $\beta$ are given by

$$
-\beta \leqslant y_{i} \leqslant \beta
$$

$$
\beta=\lambda_{\max }\left(j Z_{0}\right)
$$

and $Z_{0}$ is given by the two different cases below 
Case (i) $Q>0$ and $H \geqslant 0$

$$
Z_{0}=\frac{1}{2}\left(A^{\mathrm{T}}-Q A Q^{-1}\right)
$$

Case (ii) $Q=Q^{\mathrm{T}}$ and $H>0$

$$
Z_{0}=\frac{1}{2}\left(A-H A^{\mathrm{T}} H^{-1}\right)
$$

$\triangle$

Proof

Case (i) $Q>0$ and $H \geqslant 0$

Di Ruscio proved that the imaginary parts of the closed-loop eigenvalues are bounded when $H>0$, irrespective of how the state weight matrix $Q=Q^{\mathrm{T}}$ is chosen, provided there is a real symmetric solution of the ARE.

Here we will prove that a corresponding result exists for the case where $H \geqslant 0$ and $Q>0$. When $Q>0$ then the imaginary parts of the closed-loop eigenvalues are bounded, from up and below, irrespective of how the control input weight matrix $P>0$ is chosen. This result may be derived from the adjoint system.

Note that the adjoint equation associated with $\dot{\boldsymbol{x}}$ is

$$
\dot{p}=-\left(A^{\mathrm{T}}+Q R^{-1}\right) p
$$

and that the ARE, Equation (5), can be written as

$$
(A-H R)=-R^{-1}\left(A^{\mathrm{T}}+Q R^{-1}\right) R
$$

and that the closed-loop eigenvalues can be computed from the adjoint system matrix, that is

$$
\lambda(A-H R)=-\lambda\left(A^{\mathrm{T}}+Q R^{-1}\right)
$$

When $Q>0$, ( $Q$ non-singular), then we have the following equality

$$
Q^{-1}\left(A^{\mathrm{T}}+Q R^{-1}\right)-\left(A^{\mathrm{T}}+Q R^{-1}\right)^{\mathrm{T}} Q^{-1}=Q^{-1} A^{\mathrm{T}}-A Q^{-1}
$$

Note that both terms, on the left and right sides of Eqn (14), are skew-symmetric, and that the eigenvalues of a skew-symmetric matrix are purely imaginary.

Let $s=x-j y$ be an eigenvalue and $v$ a right eigenvector of the adjoint system matrix $\left(A^{\mathrm{T}}+Q R^{-1}\right)$. Substituting $v$ and $s$ into (14) gives

$$
\left(s-s^{*}\right) v^{*} Q^{-1} v=v^{*}\left(Q^{-1} A^{\mathrm{T}}-A Q^{-1}\right) v
$$

or

$$
-2 y j=\frac{v^{*}\left(Q^{-1} A^{\mathrm{T}}-A Q^{-1}\right) v}{v^{*} Q^{-1} v}
$$

Eqn (16) can be written

$$
y=\frac{v^{*}\left[j Q^{-1} \frac{1}{2}\left(A^{\mathrm{T}}-Q A Q^{-1}\right)\right] v}{v^{*} Q^{-1} v}
$$

Note that the matrix in the nominator of Eqn (17) is Hermitian, and that the eigenvalues of a Hermitian matrix are real. We use the inequality connecting ratios of quadratic forms, Lemma A2 Appendix, and get

$$
\lambda_{\min }\left[j \frac{1}{2}\left(A^{\mathrm{T}}-Q A Q^{-1}\right)\right] \leqslant y \leqslant \lambda_{\max }\left[j_{2}^{1}\left(A^{\mathrm{T}}-Q A Q^{-1}\right)\right]
$$


Let us define the upper bound as

$$
\beta=\lambda_{\max }\left[j \frac{1}{2}\left(A^{\mathrm{T}}-Q A Q^{-1}\right)\right]=\lambda_{\max }\left(j Z_{0}\right)
$$

Note that the eigenvalues of $Z_{0}$ are purely imaginary, and that that the eigenvalues consists of complex conjugate pairs, because the matrix $Z_{0}$ is real. Then we must have that

$$
\lambda_{\max }\left(j Z_{0}\right)=-\lambda_{\min }\left(j Z_{0}\right)=\beta
$$

The consequence is that $y$, given by (17), is bounded by

$$
-\beta \leqslant y \leqslant \beta
$$

where $\beta$ is given by (19).

Inequality (21) must hold for all $n$ eigenvalues, and Inequality (7) is proved for the case when $Q>0$.

Case (ii) $\boldsymbol{Q}=\boldsymbol{Q}^{\mathrm{T}}$ and $H>0$

The fact that the imaginary part of the closed-loop eigenvalues is bounded when $H>0$, irrespective of how the state weight matrix $Q=Q^{\mathrm{T}}$ is chosen, provided there is a real symmetric solution of the ARE, was proved in Di Ruscio (1991). An alternative, and more direct proof, is given below.

When $H>0$, ( $H$ non-singular), we have the following equality

$$
H^{-1}(A-H R)-(A-H R)^{\mathrm{T}} H^{-1}=H^{-1} A-A^{\mathrm{T}} H^{-1}
$$

where we have assumed that $R$ is a real symmetric solution of the ARE, corresponding to a state weight matrix $\boldsymbol{Q}=\boldsymbol{Q}^{\mathrm{T}}$, not necessary positive semi-definite. See Di Ruscio (1991) for the existence of solutions to the ARE in this context.

Let $s=\boldsymbol{x}-j y$ be an eigenvalue and $\boldsymbol{v}$ a right eigenvector of the closed loop system matrix $(A-H R)$. Substituting $v$ and $s$ into (22) gives

$$
\left(s-s^{*}\right) v^{*} H^{-1} v=v^{*}\left(H^{-1} A-A^{\mathrm{T}} H^{-1}\right) v
$$

or

$$
-2 y j=\frac{v^{*}\left(H^{-1} A-A^{\mathrm{T}} H^{-1}\right) v}{v^{*} H^{-1} v}
$$

Equation (24) can be written

$$
y=\frac{v^{*}\left[j H^{-1} \frac{1}{2}\left(A-H A^{\mathrm{T}} H^{-1}\right)\right] v}{v^{*} H^{-1} v}
$$

We use the inequality connecting ratios of quadratic forms, and get

$$
\lambda_{\min }\left[j_{2}^{1}\left(A-H A^{\mathrm{T}} H^{-1}\right)\right] \leqslant y \leqslant \lambda_{\max }\left[j \frac{1}{2}\left(A-H A^{\mathrm{T}} H^{-1}\right)\right]
$$

with equality, if and only if, $Q$ is chosen according to Lemma 3 in Di Ruscio (1991). Inequality (26) must hold for all $n$ eigenvalues, and Inequality (7) is proved for the case when $\boldsymbol{H}>\mathbf{0}$.

\subsection{Magnitude of the closed loop eigenvalues}

The main result in this section is presented in the following theorem.

\section{Theorem 2}

The LQ-optimal closed-loop eigenvalues, $\left(s_{i}=x_{i}+j y_{i}, i=1, \ldots, n\right)$, are bounded by

$$
r_{1}^{2} \leqslant\left(x_{i}+\alpha\right)^{2}+y_{i}^{2} \leqslant r_{2}^{2}
$$


where $r_{1}$ and $r_{2}$ are given by

$$
\begin{aligned}
& r_{1}^{2}=\lambda_{\min }(W) \\
& r_{2}^{2}=\lambda_{\max }(W)
\end{aligned}
$$

and $W$ is given by the two different cases below

Case (i) $Q>0$ and $H \geqslant 0$

$$
W=\left((A+\alpha I) Q^{-1}(A+\alpha I)^{\mathrm{T}}+H\right) Q
$$

Case (ii) $Q=Q^{\mathrm{T}}$ and $H>0$

$$
W=\left((A+\alpha I)^{\mathrm{T}} H^{-1}(A+\alpha I)+Q\right) H
$$

$\triangle$

\section{Proof}

Case (i) $Q>0$ and $H \geqslant 0$

The ARE, Eqn (5), can be written

$$
\left(A^{\mathrm{T}}+\alpha I+Q R^{-1}\right)^{\mathrm{T}} Q^{-1}\left(A^{\mathrm{T}}+\alpha I+Q R^{-1}\right)=(A+\alpha I) Q^{-1}(A+\alpha I)^{\mathrm{T}}+H
$$

From (32) we have

$$
\begin{aligned}
\left(A^{\mathrm{T}}+Q R^{-1}\right)^{\mathrm{T}} Q^{-1}\left(A^{\mathrm{T}}\right. & \left.+Q R^{-1}\right)+\alpha\left[\left(A^{\mathrm{T}}+Q R^{-1}\right)^{\mathrm{T}} Q^{-1}\right. \\
& \left.+Q^{-1}\left(A^{\mathrm{T}}+Q R^{-1}\right)\right]+\alpha^{2} Q^{-1}=(A+\alpha I)^{\mathrm{T}} Q^{-1}(A+\alpha I)+H
\end{aligned}
$$

Let $s=x+j y$ be an eigenvalue and $v$ a right eigenvector of the adjoint system matrix $\left(A^{\mathrm{T}}+Q R^{-1}\right)$. Substituting $v$ and $s$ into (33) gives

$$
\left(s^{*} s+\alpha\left(s^{*}+s\right)+\alpha^{2}\right) v^{*} Q^{-1} v=v^{*}\left[(A+\alpha I) Q^{-1}(A+\alpha I)^{\mathrm{T}}+H\right] v
$$

or

$$
x^{2}+2 \alpha x+\alpha^{2}+y^{2}=\frac{v^{*}\left[(A+\alpha I) Q^{-1}(A+\alpha I)^{\mathrm{T}}+H\right] v}{v^{*} Q^{-1} v}
$$

We use the inequality connecting ratios of quadratic forms, Lemma A2 Appendix, and get

$$
\lambda_{\min }(W) \leqslant(x+\alpha)^{2}+y^{2} \leqslant \lambda_{\max }(W)
$$

where

$$
W=\left[(A+\alpha I) Q^{-1}(A+\alpha I)^{\mathrm{T}}+H\right] Q
$$

(36) must hold for all $n$ eigenvalues, and Inequality (27) is proved for the case when $Q>0$.

Case (ii) $Q=Q^{\mathrm{T}}$ and $\mathrm{H}>0$

The ARE, Eqn. (5), can be written

$$
(A+\alpha I-H R)^{\mathrm{T}} H^{-1}(A+\alpha I-H R)=(A+\alpha I)^{\mathrm{T}} H^{-1}(A+\alpha I)+Q
$$

From (38) we have

$$
\begin{aligned}
(A-H R)^{\mathrm{T}} H^{-1}(A-H R)+\alpha\left[(A-H R)^{\mathrm{T}} H^{-1}+H^{-1}(A-H R)\right]+\alpha^{2} H^{-1} \\
=(A+\alpha I)^{\mathrm{T}} H^{-1}(A+\alpha I)+Q
\end{aligned}
$$


Let $s=x+j y$ be an eigenvalue and $v$ a right eigenvector of the closed loop system matrix $(A-H R)$. Substituting $v$ and $s$ into (39) gives

$$
\left(s^{*} s+\alpha\left(s^{*}+s\right)+\alpha^{2}\right) v^{*} H^{-1} v=v^{*}\left[(A+\alpha I)^{\mathrm{T}} H^{-1}(A+\alpha I)+Q\right] v
$$

or

$$
x^{2}+2 \alpha x+\alpha^{2}+y^{2}=\frac{v^{*}\left[(A+\alpha I)^{\mathrm{T}} H^{-1}(A+\alpha I)+Q\right] v}{v^{*} H^{-1} v}
$$

We use the inequality connecting ratios of quadratic forms, and get

$$
\lambda_{\min }(W) \leqslant(x+\alpha)^{2}+y^{2} \leqslant \lambda_{\max }(W)
$$

where

$$
W=\left[(A+\alpha I)^{\mathrm{T}} H^{-1}(A+\alpha I)+Q\right] H
$$

(42) must hold for all $n$ eigenvalues, and Inequality (27) is proved for the case when $H>0$.

We end this section by presenting two lemmas which relate the closed-loop poles to $\mathrm{A}, \mathrm{H}$ and $\mathbf{Q}$.

\section{Lemma 1}

Let $s_{i}, i=1, \ldots, n$ be the closed-loop eigenvalues, and $Q>0$, then we have

$$
\begin{aligned}
{\left[\prod_{i=1}^{n} s_{i}\right]^{2} } & =[\operatorname{det}(A-H R)]^{2}=\frac{\operatorname{det}\left(A Q^{-1} A^{\mathrm{T}}+H\right)}{\operatorname{det}\left(Q^{-1}\right)} \\
& =\operatorname{det}\left(A Q^{-1} A^{\mathrm{T}} Q+H Q\right)
\end{aligned}
$$

which relates the closed-loop poles to $\mathrm{A}, \mathrm{H}$ and $\mathrm{Q}$.

$\triangle$

\section{Lemma 2}

Let $s_{i}, i=1, \ldots, n$ be the closed-loop eigenvalues, and $H>0$, we have

$$
\begin{aligned}
{\left[\prod_{i=1}^{n} s_{i}\right]^{2} } & =[\operatorname{det}(A-H R)]^{2}=\frac{\operatorname{det}\left(A Q^{-1} A^{\mathrm{T}}+H\right)}{\operatorname{det}\left(Q^{-1}\right)} \\
& =\operatorname{det}\left(H A^{\mathrm{T}} H^{-1} A+H Q\right)
\end{aligned}
$$

which relates the closed-loop poles to $\mathrm{A}, \mathrm{H}$ and $\mathrm{Q}$.

$\triangle$

\section{Proof of Lemmas 1 and 2}

Take the determinant on both sides of Eqns (32) and (38) with $\alpha=0$. QED.

Note that the geometric mean of the closed-loop eigenvalues may be computed from the relations in Lemmas 1 and 2.

\subsection{Real parts of the closed loop eigenvalues}

Theorem 3 (Anderson and Moore, 1969)

The real parts of the LQ-optimal closed-loop eigenvalues, $\left(s_{i}=x_{i}+j y_{i}, i=1, \ldots, n\right)$ are bounded by

$$
x_{i}<-\alpha
$$


The fact that the real parts of the closed-loop eigenvalues are located to the left of a line $-\alpha$ in the left half of the $s$ plane is proved in Anderson and Moore (1969). A different proof will be given below, because it will lead us to a useful result.

Let $s=x+j y$ be an eigenvalue and $v$ a right eigenvector of the closed-loop system matrix $(A-H R)$, then

$$
(A-H R) v=s v, v^{*}(A-H R)^{\mathrm{T}}=s^{*} v^{*}
$$

Pre-multiplication with $v^{*}$ and post-multiplication with $v$ in the ARE give

$$
\left[(A-H R)^{\mathrm{T}} R+R(A-H R)+2 \alpha R+R H R+Q\right) v=0
$$

where $\alpha \geqslant 0$ is the prescribed degree of stability. Substituting (47) into (48) gives

$$
\left(s^{*}+s+2 \alpha\right) v^{*} R v=-v^{*}(Q+R H R) v
$$

Since $Q \geqslant 0$, and $R \geqslant 0$ is guaranteed from the ARE, then we have from (49) that

$$
s^{*}+s+2 \alpha=2 x+2 \alpha<0 \Rightarrow x<-\alpha
$$

(50) must hold for all $n$ eigenvalues, and Inequality (46) is proved.

If the inequality connecting the ratio of two quadratic forms, see Appendix Lemma A2, is used on Eqn (49) with $R>0$, then we have that the real parts of the closed-loop eigenvalues are bounded, from up and below, by the result in the following theorem.

\section{Theorem 4}

Let $Q \geqslant 0$ and $H \geqslant 0$. The real parts of the closed-loop eigenvalues, $\left(s_{i}=x_{i}+j y_{i}\right.$, $i=1, \ldots, n)$, are bounded, from up and below, by

$$
-\frac{1}{2} \lambda_{\max }\left(Q R^{-1}+R H\right) \leqslant x_{i}+\alpha \leqslant-\frac{1}{2} \lambda_{\min }\left(Q R^{-1}+R H\right)
$$

where $R$ is the maximum solution of the ARE (5), assumed to be positive definite.

$\triangle$

Inequality (51) states how far the eigenvalues are from $\alpha$ stability, i.e. the line $-\alpha, \alpha \geqslant 0$. Any general relation which does not involve the solution $R$ of the ARE, which only require the matrices $A, H$ and $Q$, is not known at the present.

Equations (7) and (9) show that $Q$ can be used to design acceptable bounds on the imaginary parts of the closed-loop eigenvalues. From Eqn (9) we have

$$
2 Z_{0}=\left(A^{\mathrm{T}} Q-Q A\right) Q^{-1}
$$

This means in the extreme case, that an LQ-optimal system, when $Q>0$, has only real eigenvalues, for all $P>0$, if the right-hand side of Eqn (52) is zero. This leads us to the following lemma.

\section{Lemma 3}

The closed-loop eigenspectrum is given by

if

$$
\lambda(A+B G)=-\left(\lambda\left((A+\alpha)^{2}+Q H\right)\right)^{1 / 2}
$$

or

$$
A^{\mathrm{T}} Q-Q A=0
$$

$$
A H-H A^{\mathrm{T}}=\mathbf{0}
$$

provided there is a real symmetric solution of the ARE. 


\section{Proof}

Let $F$ be the Hamiltonian matrix of the state and adjoint system, then we have

$$
F^{2}=\left[\begin{array}{cc}
A^{2}+H Q & H A^{\mathrm{T}}-A H \\
A^{\mathrm{T}} Q-Q A & \left(A^{2}+H Q\right)^{\mathrm{T}}
\end{array}\right] \in R^{2 n \times 2 n}
$$

Substituting (54) or (55) into the off diagonal matrix elements of (56) and comparing the eigenvalues, prove the lemma. QED.

If the Conditions (54) and (55) are not satisfied, then Eqn (53) may give a good approximation of the closed-loop spectrum. However, in this case, the error in using (53) should be investigated, for example by (27).

Once $Q$ has been chosen, $P$ can be used to design the required stability margin, i.e. the distance of the real parts of the closed-loop eigenvalues to the imaginary axis. The closed-loop poles are located to the left of the line $-\alpha \leqslant 0$. How far they are from this line can be predicted from (7) and (27). We have that the real parts of the eigenvalues are bounded by

$$
r_{1}^{2}-\beta^{2} \leqslant\left(x_{i}+\alpha\right)^{2} \leqslant r_{2}^{2}-\beta^{2} \leqslant r_{2}^{2}
$$

where

$$
\beta^{2}=\lambda_{\max }\left(-Z_{0}^{2}\right)
$$

$r_{1}, r_{2}$ and $Z_{0}$ are explicitly given by the matrices $A, B, P$ and $Q$, see Theorems 1 and 2 .

\section{Concluding remarks}

It has been shown that the closed-loop eigenvalues of an LQ-optimal system are located inside the region bounded by two half circles with radii $r_{1}$ and $r_{2}$ and centre at $-\alpha$, and two lines parallel to the negative real axis. This region is illustrated in Figure (1).

\section{REFERENCES}

ANDERSON, B. D. O., and MOORE, J. B.,(1969). Linear system optimization with prescribed degree of stability, Proc. IEE, 116, 2083-2087.

Di RuSCIO, D. and BALCHEN, J. G. (1990). A Schur method for designing LQ-optimal systems with prescribed eigenvalues, Modeling, Identification and Control, 11, 55-72.

Di Ruscio, D. (1991). Maximal imaginary eigenvalues in optimal systems, Proc. 1th ECCEuropean Control Conference, Grenoble, July 2-5.

Kalman, R. E. and BerTram, J. E. (1960). Control System Analysis and Design Via the 'Second Method' of Lyapunov, Journal of Basic Engineering, 82, 371-393.

KUČERA, V. (1989). Algebraic Riccati equation: Symmetric and definite solutions, Workshop on The Riccati equation in control systems, and signals, Como, Italy, 1989. With the participation of IEEE-Control Systems Society. Printed by Pitagora Editrice Bologna, Via del Legatore 3, Bologna.

LANCASTER, P. and RODMAN, L. (1980). Existence and uniqueness theorems for the algebraic Riccati equation, Int. J. Control, 32, 285-309.

LaUB, A. J. (1979). A Schur method for Solving Algebraic Riccati Equations, IEEE Trans. Automatic Control, 24, 913-921.

MAGNUS, J. R. and NEUDECKER, H. (1988). Matrix differential calculus with applications in statistics and econometrics (John Wiley and Sons, Chichester).

Willems, J. C. (1971). Least Squares Optimal Stationary Control and the Algebraic Riccati Equation, IEEE Trans. Automatic Control, 16, 621-634. 


\section{Appendix}

The Rayleigh principle is stated in the following lemma. A proof is given in Magnus and Neudecker (1988) pp. 203.

\section{Lemma A1}

For any real symmetric $n \times n$ matrix $Q$,

$$
\lambda_{\min }(Q) \leqslant \frac{x^{\mathrm{T}} Q x}{x^{\mathrm{T}} x} \leqslant \lambda_{\max }(Q)
$$

$\triangle$

The lemma bounds the ratio of two quadratic forms.

\section{Lemma $A 2$}

Let $\boldsymbol{Q}$ be a symmetric matrix, and $\boldsymbol{P}$ a positive definite matrix, then we have the following inequality connecting the ratio of two quadratic forms

$\triangle$

$$
\lambda_{\min }\left(Q P^{-1}\right) \leqslant \frac{x^{\mathrm{T}} Q x}{x^{\mathrm{T}} P x} \leqslant \lambda_{\max }\left(Q P^{-1}\right)
$$

\section{Proof of Lemma A2}

Inequality (60) was proved in Kalman and Betram (1960) for the case where both $Q$ and $\boldsymbol{P}$ are positive definite.

Here we will prove that the relations in Lemma A2, holds for any symmetric matrix $\boldsymbol{Q}$. Extension to the Hermitian (complex) case is straightforward.

The matrix $P$ is assumed to be positive definite, then the (Cholesky) decomposition exists. We have

$$
P=L^{\mathrm{T}} L
$$

where $L \in \boldsymbol{R}^{n \times n}$ is a lower triangular matrix. Change coordinate system with $z=L x$, and substitute into the ratio of quadratic forms. We have

$$
\frac{x^{\mathrm{T}} Q x}{\boldsymbol{x}^{\mathrm{T}} \boldsymbol{P x}}=\frac{z^{\mathbf{T}} L^{-\mathbf{T}} Q L^{-1} z}{z^{\mathbf{T}} z}
$$

From the Rayleigh quotient inequality, Lemma A1, we have

$$
\lambda_{\min }\left(L^{-\mathrm{T}} Q L^{-1}\right) \leqslant \frac{z^{\mathrm{T}} L^{-\mathrm{T}} Q L^{-1} z}{z^{\mathrm{T}} z} \leqslant \lambda_{\max }\left(L^{-\mathrm{T}} Q L^{-1}\right)
$$

Use the fact that, for any $n \times n$ matrices $A$ and $B$,

$$
\lambda_{i}(A B)=\lambda_{i}(B A), \quad \forall i=1, \ldots, n
$$

and the relations in (60) are proved. 